\title{
Molecular Pathogenesis of Atherosclerosis and Implication for Therapy
}

Jeanne APa

aDepartment of Histology, Faculty of Medicine, University of Indonesia, Jl. Salemba 6, Jakarta Pusat, Jakarta, 10430, Indonesia

\section{ABSTRACT}

A growing body of evidence supports the role of inflammation in the pathogenesis of atherosclerosis. However, the supposed initiation factors of atherogenesis are infection and change in shear stress on certain location, leading to attachment of LDL and subsequent oxidation. The pathway activated are the NFkB and TGFB leading to endothelial dysfunction and production of inflammatory cytokines and adhesion factors followed by recruitment of inflammatory cells to the site, oxLDL internalization and foam cell formation in the fatty streak that later develop into atherosclerotic plaque. Further, p53signaling causes apoptosis leading to plaque rupture, platelet activation and aggregation ending in clinical manifestations. Moreover, numerous individual risk factors might aggravate the condition, and the progress might take decades depending on the balance of pro and anti atherogenic factors. Therefore, management of atherosclerosis addressing the individual risk factors using drugs with various properties coping with the molecular basis especially inflammation is beneficial.

\section{KEYWORDS: Leukocyte trafficking, intima inflammation, fatty streak, foam cells}

\section{INTRODUCTION}

Recently, a growing body of evidence supports the role of chronic inflammation in the pathogenesis of atherosclerosis. The evidence showed that atheroma plaque contains $\mathrm{T}$ lymphocytes that are part of $\mathrm{Ag}$ specific/adaptive immune response, and mononuclear phagocytic lineage that are part of the innate immune response. ${ }^{1}$ The $T$ lymphocytes present are especially from the pro inflammatory Th1 type that is pro atherogenic, though the antigen specificity is not well established. A fraction is reactive to oxidized LDL (oxLDL), modified LDL molecules, or other antigens.

Many risk factors are attributed to atherosclerosis, but mostly are systemic, such as chronic infection, hypertension, lipid abnormalities, obesity, especially intra abdominal l/ visceral/ metabolic obesity, diabetes, autoimmunity, or elevated levels of plasma factors such as fibrinogen, etc. ${ }^{2-9}$ while atherosclerosis occurred only on certain sites. Atherosclerosis is believed to be preceded by initial endothelial inflammation aggravated by other atherogenic risk factors. However, the predilection sites of atherosclerosis such as at branching and bending points pose questions of how the systemic risk factors lead to development of atheroma plaques only at certain site and not the en-

Corresponding author

Jeane Adiwinata Pawitan

Department of Histology, Faculty of Medicine,

University of Indonesia, Jakarta, Indonesia

e-mail: jeanneadiwip@fk.ui.ac.id or

jeanneadiwip@gmail.com tire blood vessel. This review addresses the development of atherosclerosis, especially factors that lead to molecular events, including the role of chronic infection, haemodynamic factors (shear stress), modified LDL, and the various subsequent impairments in the level of molecular factors. Finally, implication to therapy is addressed.

\section{Development of atherosclerosis}

Atherosclerosis begins with uptake of modified LDL at certain sites followed by accumulation, adhesion and penetration of inflammatory cells especially macrophages and lymphocytes into the intima of blood vessels leading to the formation of fatty streaks. The fatty streak then develops micro vessels containing atheroma plaque. Therefore an atheroma plaque has macro and microvasculature surfaces. The whole process continues on the macro and microvasculature, and then aggravated by smooth muscles proliferation and migration to the intima followed by extra cellular matrix formation that after a long period that usually takes decades ends up in fibrous atheromatous lesion/atherosclerotic plaque that is prone to rupture leading to thrombosis that provokes the clinical manifestations (Figure 1). ${ }^{5}$

The role of chronic infection in atherogenesis

Infection both viral such as by herpes and cytomegalo viruses and bacterial such as by Chlamydia and Helicobacter species was thought to play a role in atherogenesis and acute coronary syndrome. Herpes viruses, Chlamydia penumoniae and Helicobacter pylori DNA was found in human atheroma, ${ }^{5-7}$ though 
Helicobacter pylori and Chlamydia penumoniae DNA was also found in non atherosclerotic control arteries without significant difference. ${ }^{5,6}$ Therefore, infection might serve as initiation of atherogenesis that takes time to develop, thus in the early process, the bacteria can be found in normal seeming arteries.

Two studies showed correlation between Chlamydia pneumoniae and Helicobacter pylori with atherogenesis due to the presence of Helicobacter pylori and Chlamydia penumoniae DNA along with interleukin-6 (IL-6), ${ }^{6}$ that induces the liver to produce high titers of acute phase reactants i.e. C-reactive protein (CRP). ${ }^{6,7}$ Further, the presence of Chlamydia pneumoniae Ig G was correlated with the risk of developing coronary heart disease. ${ }^{8}$ Another study showed that $85.5 \%$ of continuous ambulatory peritoneal dialysis patients had poor oral health status and periodontal disease. In addition, periodontal disease was correlated to markers of inflammation, and atherosclerosis. ${ }^{10}$ Furthermore, a systematic review and meta-analysis showed that periodontal disease with elevated bacterial exposure was correlated to carotid intima-media thickening indicating early atherogenesis. ${ }^{11}$

\section{The role of hypertension in atherogenesis}

Hypertension might be due to many factors such as increase activity in renin angiotensin system or other factors, ${ }^{12}$ and subsequently, hypertension causes increase in haemodynamic forces (Figure 1).

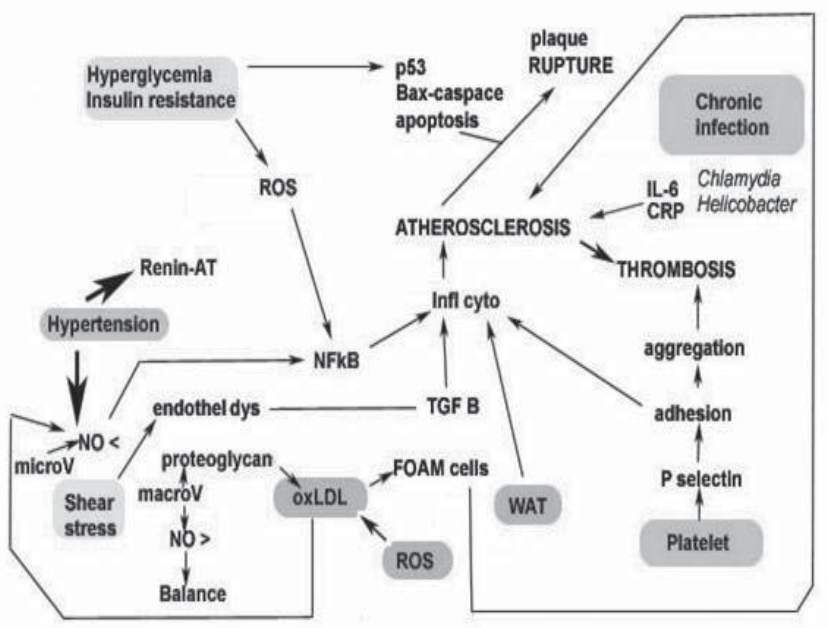

Figure 1. Pathogenesis of atherosclerosis

Renin-AT = renin - angiotensin system, NO < = decreased nitric oxide, micro $\mathrm{V}$ = microvasculature, endothel dys = endothelial dysfunction, macro $\mathrm{V}=$ macrovasculature, $\mathrm{NO}>$ = increased nitric oxide, NFKB = nuclear factor kappa $B$, ROS = reactive oxygen species, infl cyto = inflammatory cytokines, TGF B= transforming growth factor beta, WAT = white adipose tissue, IL-6 = interleukin $6, \mathrm{CRP}=\mathrm{C}$ reactive protein.

\section{The role of haemodynamic forces in atherogenesis}

Haemodynamic forces cause local shearing forces leading to shear stress on endothelium of blood ves- sel wall, and the stress was unevenly distributed. The endothelium lining the blood vessel was proven to be sensitive, as a certain amount of laminar shear stress given to cultured endothelium for 96 hours caused actin polymerization at sites where stress fibers were inserted into focal adhesion complexes causing stress fiber assembly and realignment, ${ }^{13}$ causing a change in cytoskeleton and elongation of the endothelial cell in the direction of the stress. ${ }^{13,14}$ The sensitivity to threshold-exceeding shear stress was thought to impair endothelium functions causing endothelial dysfunction that showed pro atherogenic response due to up regulation of endothelial expressions of endothelin, adhesion molecules such as vascular cell adhesion molecule-1 (VCAM-1) and inter-cellular adhesion molecule-1 (ICAM-1), and various cytokines and growth factors such as platelet-derived growth factors (PDGF) and transforming growth factor-B1 (TGF-B1). One of the various cytokines is the chemokine (chemotactic cytokine) called monocyte chemo-attractant proteins (MCP-1)/CCL2, which recruit monocytes that migrate into the intima and become macrophages. ${ }^{13,15,16}$

Further, macrophages and smooth muscles produce more pro-inflammatory cytokines: i.e. interleukin-8 (IL-8), interferon $\gamma$ inducible protein 10 (IP10), more monocyte chemoattractant proteins (MCP-1, MCP-4), and interferon inducible $T$ cell $\alpha$-chemoattractant (ITAC) leading to chemotaxis of inflammatory cells. ${ }^{5}$

Higher pulsating shear stress on certain site that might be due to increased blood pressure also causes an increase in endothelial nitric oxide synthase (eNOS) gene production and thus nitric oxide (NO), while low shear stress causes an opposite response. Nitric oxide has anti atherogenic properties, and increased NO production subsequently causes vasodilation that is intended to control the blood pressure. ${ }^{17}$

Upregulation of ICAM-1, PDGF and eNOS is thought under the influence of transcription factors of the rel family. ${ }^{5}$ However, one of the member of the rel family member is the shear stress regulated nuclear factor kappa B (NFkB), ${ }^{18,19}$ whose activation is due to reduced shear stress and subsequent decrease in NO production in microvasculature. ${ }^{5}$

Further, shear stress causes the endothelial and muscle cells of the intima to express heparan sulfate proteoglycan that binds the apoprotein B100 moeity of LDL, thus making the LDL susceptible to modification especially oxidation. ${ }^{5}$ Therefore, change in shear stress on a certain site might initiate atherogenesis on that site, and might explain why atherogenesis occurs on predilection sites only and not along the entire blood vessel.

\section{The role of renin angiotensin system in atherogen- esis}

The renin-angiotensin (RAS) hormonal system controls body homeostasis of fluid, blood pressure, and cardiovascular function. Every tissue and organ has 
their own locally regulated RAS system. In this system, renin (a peptidase) cleaves angiotensinogen into angiotensin I (a mild vasodilator that is subsequently cleaved by the angiotensin-converting enzyme into the biologically active angiotensin II that is a potent vasoconstrictor). The components of RAS system might be produced locally and/or may be taken from the circulation. An overactive RAS system produces more angiotensin II leading to hypertension. In addition, it induces the generation of reactive oxygen species ROS) that causes "oxidative stress," activation of the NFkB pathway that causes production of inflammatory cytokines, and endothelial dysfunction. Further, it causes cell hypertrophy, fibroblast proliferation, excess of extra cellular matrix formation and finally atherosclerotic plaque. ${ }^{12}$

\section{The role of modified LDL in the formation of fatty streak and atheroma plaque}

Many kinds of modified LDL are thought to play a role in atherogenesis, such as oxidized LDL (oxLDL), acetylated LDL, aggregated LDL, LDL-IgG complex, LDL- proteoglycans complex that make it prone to oxidation and aggregation, and enzymatically modified LDL. ${ }^{20}$

Modified LDLs, especially oxidized LDL (oxLDL) bound by heparan sulfate proteoglycan is proven to induce pro inflammatory genes, stimulate the endothelium to express CCR2 (MCP-1 rec), and to produce MCP-1, monocyte colony stimulating factor (M-CSF), interleukin-1 (IL-1), leucocyte adhesion molecules ( $\mathrm{E}-$ selectin, P-selectin, ICAM-1, and VCAM-1), and activate $T$ cells to make auto antibodies toward oxLDL. Oxidized LDL itself is a potent chemo-attractant for inflammatory cells especially monocytes and to a certain extent for lymphocytes. . $^{2,20}$

The binding of oxLDL and production of MCP-1 attract more monocytes and lymphocytes to the area and subsequent binding of the inflammatory cells to adhesion molecules causes transmigration/ trafficking across endothelium that ends up in vascular intima inflammation. Macrophages accumulated in the intima lead to the formation of foam cell rich fatty streak. ${ }^{5}$

\section{Formation and accumulation of foam cells}

Oxidized LDL is prone to aggregation, and in the form of aggregate it is more readily taken up by macrophages via their scavenger receptor. Scavenger receptors for modified LDL (Table 1) can be found on macrophages, smooth muscles, and endothelium. One of the endothelial receptor, the lectin-like oxLDL receptor-1 (LOX-1) contains unique repetitive sequence that causes up-regulation of the receptor upon increase in blood pressure. ${ }^{21}$ Therefore, hypertension as a risk factor of atherosclerosis can be explained by this additional fact.

\begin{tabular}{|l|l|}
\hline Modified LL & Receptors \\
\hline Oxidized LL & $\begin{array}{l}\text { SRA, CD-36, CD-38 (macrosialin), LOX-1, } \\
\text { SR-PSOX }\end{array}$ \\
\hline Acetylated LD & SRA \\
\hline Aggregated LD & nativeLD-R \\
\hline LL-Ig G complex & LL-R, Fc Rec \\
\hline
\end{tabular}

Table I. Modified LDLs and their receptors ${ }^{20,21}$

$L D L=$ low density lipoprotein, $L O X-1=$ lectin-like oxidized LDL receptor-1, SR-PSOX= scavenger receptor for phosphatidylserine and oxidized lipoprotein, $L D L-R=$ low density lipoprotein receptor

When the cells contain a lot of oxLDL, they transform into foam cells. Further, oxLDL stimulates macrophages to express more scavenger receptor, thus more oxLDL uptake, and inhibits macrophage motility, thus causing the macrophages to remain at the site of atherogenesis. In addition, ox LDL confers mitogenic activity to smooth muscle cells and macrophages, causing them to accumulate at the site of atherogenesis and becoming the sources of foam cells. Further, macrophages secrete growth factors that cause evolution of the fatty streak due to stromal cell proliferation, extra-cellular matrix remodeling, and neovascularization that ends up in granuloma formation, thus developing surfaces of macro and microvasculature in an atheroma plaque (Figure 2). ${ }^{5,20}$

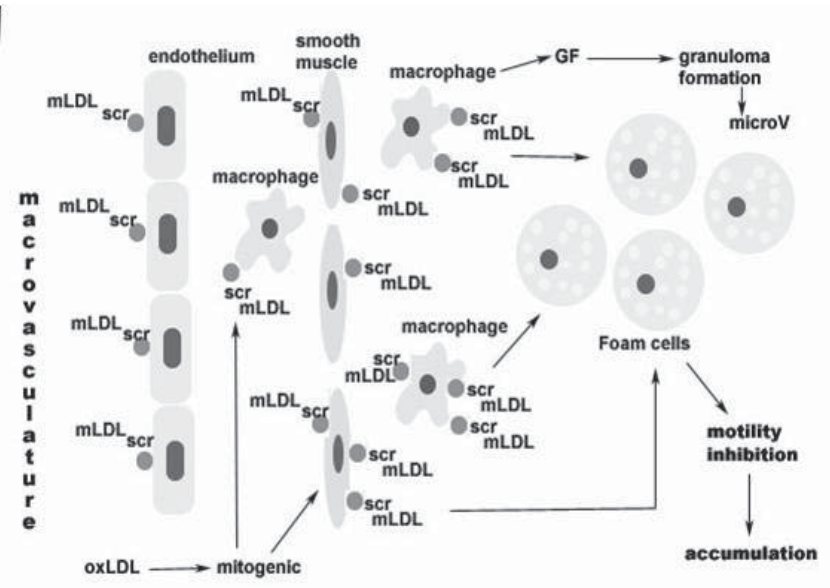

Figure 2. Foam cell and granuloma formation

$\mathrm{mLDL}=$ modified $\mathrm{LDL}, \mathrm{scr}=$ scavenger receptor, $\mathrm{oxLDL}=$ oxidized LDL, GF = growth factor, microV = micro vasculature

\section{The role of white adipose tissue in atherogenesis}

Various studies revealed that white adipose tissue secretes pro inflammatory cytokines (Figure 1) such tumor necrosis factor a (TNF-a), IL-1, interleukin-1 receptor antagonist (IL-1Ra), IL-6, IL-8, MCP-1, IP-10, RANTES, and peptides with hormonal activity such as adiponectin, leptin and resistin. Therefore, the increase in pro atherogenic cytokines secreted by the 
voluminous perivascular adipose tissue in obese individuals might exert paracrine signaling on the surrounded artery and thus contribute to inflammatory changes and thus atherogenesis. ${ }^{9}$

Further, visceral fat are different from subcutaneousfat in the properties of their resident macrophages. In visceral fat, the resident macrophages produce more of certain pro inflammatory cytokines i.e. tumor necrosis factor a (TNF-a) and interleukin-6 (IL-6), but less adiponectin. The increased cytokines might induce insulin resistance that together contribute to endothelial dysfunction and subsequent atherosclerosis in individuals with metabolic obesity. ${ }^{22}$

\section{The role of hyperglycemia and insulin resistance in atherogenesis}

A study showed that high D-glucose activated the bax-caspase pathway leading to apoptosis in human endothelium that initiated atherogenesis. Further, hyperglycemia activated the protein kinase $C$ pathway and subsequently NADPH oxidase, which was supposed as a major source of super-oxide, one of the reactive oxygen species (ROS) in cardiovascular cells. Therefore, hyperglycemia might indirectly cause oxidative stress leading to endothelial dysfunction of the coronary arteries in diabetic patients. ${ }^{23}$ Further, insulin is thought to stimulate the release of NO from endothelium. ${ }^{24}$ Therefore insulin resistance in type 2 diabetes might cause endothelial dysfunction, leading to atherogenesis (Figure 1). ${ }^{25}$

\section{The role of platelet in atherogenesis}

A study revealed that in diabetic patients, circulating $\mathrm{P}$-selectin positive platelets are higher compared to those in non-diabetic patients, and are significantly correlated to carotid atherosclerosis. Furthermore, several studies in atherosclerosis prone mice revealed that activated platelets aggregated with leukocytes, release pro inflammatory cytokines and growth regulatory molecules, causing endothelial activation, inflammatory cells recruitment and altered smooth muscle cell function leading to atherosclerosis. Further, in the circulation of patients with coronary artery diseases, activated platelets and their aggregates with leukocytes are found. ${ }^{26}$

\section{The role oxidative stress and ROS in atherogen- esis}

Reactive oxygen species are always generated in metabolic pathway, and our body has a mean to cope with normal levels of ROS. In case of increased ROS that eluded body surveillance, it might cause oxidative stress ending is various deleterious consequences, either by acting directly on various tissues and molecules such as oxidation of LDL to form oxLDL or through NO depletion, both are known to be pro atherogenic. Further, ROS modulate cell contraction, migration, growth/apoptosis and extracellular matrix turnover, in a deleterious way contributing to atherogenesis. ${ }^{27}$
Progression of fatty streak and atheroma plaque into fibrous atheromatous lesion/atherosclerotic plaque

After granuloma formation, trafficking of inflammatory cells continues, with ICAM, and P-selectin as adhesion molecules at the macro-vasculature, and VCAM as the adhesion molecule at the micro-vasculature. ${ }^{5}$ In microvasculature, decrease blood flow causes decrease in shear stress, thus decrease in NO production. Decreased NO released its inhibitory effect on NFkB, which causes up-regulation in NFkB regulated genes. In addition, decreased $\mathrm{NO}$ release the inhibitory effect on smooth muscle contraction and proliferation, on platelet aggregation, and VCAM- 1 transcription causing subsequent increase in VCAM-1. ${ }^{5}$ Further, growth factors from macrophages cause smooth muscles proliferation that migrate into intima and produce extra cellular matrix especially collagen type I.., 28

In macrovasculature, increased NO due to thresholdexceeding shear stress has anti atherogenic properties and will balance the pro atherogenic effect of other factors released by increased shear stress. Therefore, the progress of atherogenesis into fibrous atheromatous lesion/atherosclerotic plaque might take decades depending the balancing between anti and pro atherogenic properties (Figure 1).

Activation of NFkB in microvasculature and TGF-B pathway in macrovasculature ${ }^{5}$ lead to induction of various pro inflammatory cytokines (Figure 1 ), such as CXCL-2, -3, -6, -10, interleukin-8, fractalkine (CX3$\mathrm{CL} 1$ ), and regulated on activation normal $\mathrm{T}$ cell expressed and secreted [RANTES (CCL5)]. Some of them show leukocyte chemotactic properties, while others are endothelial adhesive proteins for leukocytes, thus causing accumulation of mononuclear leucocytes. Activation of NFkB pathway can also be activated by various systemic stimuli such as oxLDL, $C$ reactive protein, and cytokines such as interleukin-1, tumor necrosis factor (TNF) and several pathway proteins such as RelA, TP53BP2, TRAF1, and TRAF5. ${ }^{2}$

A study on micro-array gene studies to reveal endothelial transcriptomes/enriched gene expression in atherosclerotic lesion versus plaque free region concluded that the various chemokines, cytokines, adhesion molecules, and other molecules involved in atherosclerosis were under the NFkB and TGFB pathway, and p53signaling, which was in agreement with immunohistochemistry staining. Further, in vitro TGFB was shown to increase endothelial permeability through SMAD2-dependent p38 activation, and to induce pro atherogenic genes such as LOX-1, atherothrombotic factor PAI-1, and leukocyte adhesive proteins ICAM-1 and MCP-1. However, an opposite result was shown in a mouse model, where disruption of TGFB induced ICAM-1 and VCAM-1, showing anti-atherogenic properties of TGFB. ${ }^{2}$

In advanced atherosclerosis, soluble chemokines for a variety of leukocytes under NFkB2 pathway, phos- 
phorilated SMAD2 and ICAM-1 in TGFB pathway, and $\mathrm{BAX}$ in apoptosis related $\mathrm{p} 53$ signaling, were up regulated, while in early atherosclerosis fractalkine/CX3CL1, IP10/CCL10, which were endothelial associated cytokines for monocytes and $\mathrm{T}$ cells adhesion, and TBX18 and ICAM-1 were upregulated. However, the best marker indicating early atherosclerosis is fraxtalkine. $^{2}$

\section{Rupture of atherosclerotic plaque and clinical manisfestation}

In advanced atherosclerosis, oxLDL that is cytotoxic will cause apoptosis of the cells causing rupture of the plague, and may activate pro coagulant pathway, thus induces tissue factors and causes platelet aggregation leading to thrombosis. In addition, the macrophages secrete enzymes such as collagenase, gelatinase, stromelysin, proteinase cathepsin that cause matrix degradation/ endothelial cell desquamation leading to plaque rupture, exposing the basement membrane collagen, thus promoting platelet activation and aggregation leading to thrombosis and clinical manifestation i.e. acute coronary syndrome. ${ }^{5,29}$

Chronic inflammatory cells accumulated in atherosclerotic plaque were macrophages and lymphocytes. Among the lymphocytes, recently, two subsets different from Th1 and Th2 cells, namely CD4+CD25+Foxp3+ regulatory $\mathrm{T}$ (Treg) cells and Th17 cells were described. They have an opposite effects on autoimmunity compared to Th1 and Th2. A study showed that balance of Th17/Treg was important to prevent the progress of atherosclerotic plaque, and increase in Th17 and Th17 related cytokines and transcription factor together with decrease in Treg and Treg related cytokines and transcription factor was supposed to lead to the onset of acute coronary syndrome. ${ }^{30}$

In a majority of Japanese patients, arterial spasm is supposed to play a role in the clinical manifestation of coronary atherosclerosis, as the spasm can cause occlusion of the arterial lumen already narrowed by the presence of atherosclerotic lesion in the absence of plaque rupture. ${ }^{31}$

\section{Implication for therapy}

Efforts to delay the progress and thus clinical manifestation of atherosclerosis are aimed at the risk factors such as controlling metabolic obesity, hypertension, diabetes, lipid profile, combating chronic infection, anti platelet agents, or vasodilators.

\section{Controlling metabolic obesity and diabetes}

Controlling obesity by dieting and exercise might be beneficial to prevent or to delay the progress atherosclerosis. Further, reducing peripheral fat by $\mathrm{li}$ posuction was shown to decrease cardiovascular risk factors. However, how much visceral fat reduction needed that lead to favorable metabolic changes is still a question. ${ }^{22}$
Controlling diabetes is thought to be the best way in improving endothelial function, and thus slowing the progress of atherosclerosis in diabetic patients. Further, various trials showed that statins (usually used in controlling lipid profile) or angiotensin converting enzyme inhibitors (usually used to control hypertension) was correlated with endothelial function improvements in diabetic individuals. ${ }^{25}$

\section{Controlling hypertension}

As hypertension is one of the many risk factors of atherosclerosis, and causes increase in shear stress in macro vasculature, controlling hypertension is crucial in prevention and to halt the progress of atherosclerosis. Of the various anti hypertensive agents, some showed beneficial effect to prevent the progress of atherosclerosis, especially those having peripheral vasodilation effect. ${ }^{31,32}$ One of the many anti hypertensive agents, the selective beta-adrenoceptor blocking agent, nevibolol, besides lowering hypertension, showed beneficial effect on endothelial dysfunction, and could normalize perfusion in a study on $36 \mathrm{pa}$ tients with primary hypertension and abnormal myocardial perfusion. ${ }^{32}$

Comparison of a calcium channel blocker, nifedipine that also has antioxidant property with either of the several angiotensin-converting enzyme (ACE) inhibitor (enalapril, lisinopril or imidapril) was done in a Japan multicenter study on Japanese hypertensive patients with concomitant coronary artery disease by quantitative coronary angiogram (QCA) analysis. The result showed that nifedipine was better than ACE inhibitor in inhibiting the progression of coronary atherosclerosis and the development of coronary artery stenosis. ${ }^{31}$

\section{Controlling lipid level}

The most deleterious of all lipids is LDL especially when it is modified by oxidation (oxLDL). Therefore lipid lowering agents are thought to be able to prevent the progress of atherosclerosis. Garlic allicor tablet showed moderate hypolipidemic and antioxidative effect at a dose of $600 \mathrm{mg}$ per day in patients with clinical signs of atherosclerosis. ${ }^{33}$

In addition, one family of the most widely prescribed cholesterol-lowering agents, the statins that work as HMG-CoA reductase inhibitors showed antioxidant effect, and is supposed to improve endothelial function in nitric oxide production. ${ }^{23}$ Several cross-sectional trials on rheumatoid arthritis patients suggested that statins have beneficial effect on endothelial dysfunction, and have anti inflammatory and anti proliferative effects as they caused improvement in plasma inflammation markers, such as C-reactive protein. ${ }^{34}$ Another studies using various imaging techniques showed that statins can stop or delay the progress of atherosclerosis and in some cases, even lead to plaque regression. ${ }^{35}$ Further, statins showed few adverse effects, thus they are relatively safe. ${ }^{34}$ 
Therefore, statins can be considered as the first-line drug for the prevention of atherosclerosis progression and its clinical manifestation. Meta-analyses including several primary and secondary intervention studies showed statins significantly reduced cardiovascular events, but the responses showed considerable inter individual variations that might be due to genetic variations in lipid and lipoprotein metabolism and genetic variation in controlling statins pharmacokinetics and pharmacodynamics. ${ }^{36}$

\section{Combating chronic infection}

The use of antibiotics to treat chronic infection in patients with atherosclerosis did not show beneficial effect on the progress of atherosclerosis. ${ }^{37}$ This might be due to the fact that infection is only one of the initiating factor of atherosclerosis. $6,7,11$ Once atherogenesis is initiated, the inflammation process on the lesion will continue, whether the infection is still there or not, though the presence of chronic infection together with other risk factors will aggravate the condition. Therefore, it is supposed that preventing infection might reduce the incidence of atherogenesis.

\section{The use of antioxidants}

Various substances with antioxidant properties, such as vitamin C, vitamin $\mathrm{E}$, and edaravone, a free radical scavenger, has been used to prevent the progress of atherosclerosis. A number of trials showed that antioxidants caused short-term improvement on endothelial function in humans, but all studies on the effect of preventive antioxidant therapy showed disappointing results. ${ }^{25}$ These facts might be due to the many factors that play a role in atherogenesis, thus giving antioxidant alone only modulate one of the many factors. In addition, oxidative stress that occurs in tissue might be better controlled by tissue/cellular derived anti oxidant such as super oxide dismutase or catalase. Therefore, treatment that enhances tissue/cellular derived antioxidant might be more beneficial.

Further, anti-hypertensive agents such as angiotensinconverting enzyme (ACE) inhibitors and angiotensin receptor blockers (ARB), and anti-hyperlipidemic agents like statins, beside their original pharmacological properties, showed anti-oxidant properties. However, clinical trial using these substances showed contradictory results on the progress of atherosclerosis. ${ }^{38}$

\section{The use of anti platelet agents}

Clinical manifestation of atherosclerosis is largely due to thrombosis. Therefore reducing the platelet stickiness using anti platelet agent is very important to prevent thrombosis. Acetylsalicylic acid (aspirin), the cheapest anti platelet agent with anti inflammatory effect and thienopyridine clopidogrel, both are widely used anti-platelet drugs that significantly reduce clinical manifestations in patients with manifest atherosclerosis. However, their impact on the progress of atherosclerosis remains controversial. A study on atherosclerotic prone mice showed that combination of acetylsalicylic acid and clopidogrel reduced thrombus formation by approximately $50 \%$ after rupture of atherosclerotic plaque in vivo, but the two agents alone did not. ${ }^{39}$

However, in patients at high risk of atherothrombotic events, clinical trials revealed that combination of clopidogrel $(75 \mathrm{mg} / \mathrm{d})$ and aspirin (75-162 mg/d) showed more or less the same effect as aspirin alone for long-term prevention of atherosclerosis clinical manifestation. In addition, in patients with presumed recent arterial cerebral ischemia, oral anticoagulant (International Normalized Ratio of 2.0-3.0) was not more effective compared to aspirin alone. However, combination of extended-release dipyridamole and aspirin was more effective than aspirin alone. A trial including more than 20,000 patients with recent (< 120 days) atherothrombotic ischemic stroke to compare the effect of clopidogrel with the combination of extended-release dipyridamole and aspirin is underway. ${ }^{40}$

\section{The use of vasodilators}

To manage clinical manifestation of atherosclerosis, vasodilators are needed, together with risk factor management. In patients with hypertension, a number of anti hypertensive agents have vasodilator effect, ${ }^{31,32}$ and adjustment of therapy should refer to the whole condition of the patient.

\section{CONCLUSION}

The most important event in atherosclerosis is chronic inflammation due to the activation the NFkB and TGFBpathway, and atheroprogression causes apoptosis due to p53signaling. Therefore management of atherosclerosis addressing the individual risk factors using drugs with various properties coping with the molecular basis especially inflammation will be beneficial.

\section{REFERENCES}

1. Hansson GK. Inflammation, atherosclerosis, coronary artery disease. N Engl J Med 2005; 352:1685-95

2. Volger OL, Fledderus JO, Kisters N, et al. Distinctive Expression of Chemokines and Transforming Growth Factor- Signaling in Human Arterial Endothelium during Atherosclerosis. Am J Pathol 2007; 171: 326-37

3. Pertovaara M, Raitala A, Juonala M, et al. Autoimmunity and atherosclerosis: functional polymorphism of PTPN22 is associated with phenotypes related to the risk of atherosclerosis. The Cardiovascular Risk in Young Finns Study. Clin Exp Immunol 2006;147: 265-9

4. Vikramadithyan RK, Hu Y, Noh HL, et al. Human aldose reductase expression accelerates 
diabetic atherosclerosis in transgenic mice. $\mathrm{J}$ Clin Invest 2005;115:2434-43

5. Libby P, Hansson GK, Pober JS. Inflammation and immunity in atherogenesis. In: Chien KR. Molecular basis of cardiovascular disease. 2nd edition. Philadelphia: Saunders; 2004. p.349-364.

6. Adiloglu AK, Ocal A, Can R, et al. Detection of Helicobacter pylori and Chlamydia pneumoniae DNA in human coronary arteries and evaluation of the results with serologic evidence of inflammation. Saudi Med J 2005; 26:1068-74

7. Iriz E, Cirak MY, Engin ED, et al. Effects of a typical pneumonia agents on progression of atherosclerosis and acute coronary syndrome. Acta Cardiol 2007; 62:593-8

8. Momenah AM, Tayeb MT, Chlamydia pneumoniae seropositivity and risk of developing coronary heart disease in Western Saudi Arabia. Saudi Med J 2005;26:1926-9

9. Meier CA, Thalmann S. White adipose tissue, inflammation and atherosclerosis. [Article in French]. Bull Acad Natl Med 2007; 191:897910

10. Cengiz MI, Bal S, Gökçay S, et al. Does periodontal disease reflect atherosclerosis in continuous ambulatory peritoneal dialysis patients? J Periodontol 2007; 78:1926-34

11. Mustapha IZ, Debrey S, Oladubu M, et al. Markers of systemic bacterial exposure in periodontal disease and cardiovascular disease risk: a systematic review and metaanalysis. J Periodontol 2007; 78:2289-302

12. Raizada V, Skipper B, Luo W, et al . Intracardiac and intrarenal renin-angiotensin systems: mechanisms of cardiovascular and renal effects. J Investig Med 2007; 55:341-59

13. Noria S, Xu F, McCue S, et al. Assembly and Reorientation of Stress Fibers Drives Morphological Changes to Endothelial Cells Exposed to Shear Stress. Am J Pathol 2004; 164: 121123

14. Noria S, Cowan DB, Gotlieb Al, et al. Transient and steady state effects of shear stress on endothelial cell adherens junctions. Circ Res 1999; 85:504-14

15. Walpola PL, Gotlieb Al, Cybulsky MI, et al. Expression of ICAM-1 and VCAM-1 and monocyte adherence in arteries exposed to altered shear stress. Arterioscler Thromb Vasc Biol 1995; 15:2-10

16. Ohno M, Cooke JP, Dzau VJ, et al. Fluid shear stress induces endothelial transforming growth factor beta-1 transcription and production. Modulation by potassium channel blockade. J Clin Invest 1995; 95:1363-9

17. Cheng $C$, van Haperen $R$, deWaard $M$, et al. Shear stress affects the intracellular distribution of eNOS: direct demonstration by a novel in vivo technique. Blood 2005; 106: 3691-8

18. Helderman F, Segers D, de Crom R, et al.
Effect of shear stress on vascular inflammation and plaque development. Curr Opin Lipidol 2007; 18:527-33

19. Partridge J, Carlsen H, Enesa K, et al. Laminar shear stress acts as a switch to regulate divergent functions of NF-\{kappa\}B in endothelial cells. FASEB J 2007; 21:3553-61

20. Tsimikas S, Glass CK, Steinberg D, et al. Lipoprotein oxidation, macrophages, immunity and atherogenesis. In: Chien KR. Molecular basis of cardiovascular disease. 2nd edition. Philadelphia: Saunders; 2004. p.385-413.

21. Nagase $M$, Hirose S, Fujita T. Unique repetitive sequence and unexpected regulation of expression of rat endothelial receptor for oxidized low-density lipoprotein (LOX-1). Biochem J 1998; 330:1417-22

22. Hamdy O, Porramatikul S, Al-Ozairi E. Metabolic obesity: the paradox between visceral and subcutaneous fat. Curr Diabetes Rev 2006; 2:367-73

23. Nakagami H, Kaneda Y, Ogihara T, et al. Endothelial dysfunction in hyperglycemia as a trigger of atherosclerosis. Curr Diabetes Rev 2005; 1:59-63

24. Duncan E, Ezzat V, Kearney M. Insulin and endothelial function: physiological environment defines effect on atherosclerotic risk. Curr Diabetes Rev 2006; 2:51-61

25. Hadi HA, Suwaidi JA. Endothelial dysfunction in diabetes mellitus. Vasc Health Risk Manag 2007; 3:853-76

26. Koyama H, Nishizawa Y. Platelet in progression of atherosclerosis: a potential target in diabetic patients. Curr Diabetes Rev 2005; 1:159-65

27. San José G, Fortuño A, Beloqui O, et al. NADPH oxidase CYBA polymorphisms, oxidative stress and cardiovascular diseases. Clin Sci 2008; 114:173-82

28. Rekhter MD, Zhang K, Narayanan AS, et al. Type I collagen gene expression in human atherosclerosis. Localization to specific plaque regions. Am J Pathol 1993; 143:163448

29. Samokhin AO, Wong A, Saftig P, et al. Role of cathepsin $\mathrm{K}$ in structural changes in brachiocephalic artery during progression of atherosclerosis in apoE-deficient mice. Atherosclerosis 2008 Feb 19 [Epub ahead of print]. PMID: 18291403

30. Cheng X, Yu X, Ding YJ, et al. The Th17/Treg imbalance in patients with acute coronary syndrome. Clin Immunol 2008 Feb 20 [Epubahead of print]. PMID: 18294918

31. Yui Y. JMIC-B study and its sub-analyses: effect of nifedipine in Japanese hypertensive patients with coronary artery disease. [Article in Japanese]. Drugs 2006; 66:25-7 Erratum in: Drugs 2007; 67:1849

32. Masoli O, Redruello M, Baliño NP, et al. Use of Nebivolol for the treatment of endothelial dysfunction in patients with hypertension: 
The EDEN registry. J Cardiovasc Pharmacol 2008; 51:202-7

33. Gromnatskii NI, Sereditskaia ZhE, Lazareva NV, et al. Effects of garlic allicor tablets on lipid metabolism and risk of fatal cardiovascular complications in patients with atherogenic dyslipoproteinemia. [Article in Russian]. Vopr Pitan 2007; 76(6):60-4

34. Paraskevas KI. Statin treatment for rheumatoid arthritis: a promising novel indication. Clin Rheumatol 2008; 27:281-87

35. Kushner PR. Can intensive statin therapy halt the progression of atherosclerosis? Recent evidence and potential implications for patient management. Prog Cardiovasc Nurs 2007; 22:207-13

36. Puccetti L, Acampa M, Auteri A. Pharmacogenetics of statins therapy. Recent Patents Cardiovasc Drug Discov 2007; 2:228-36

37. No authors listed. Infection as a risk factor in the atherosclerosis progression: current concepts and treatment opportunities. [Article in Ukrainian] Lik Sprava 2007; 3:3-13

38. Inagi R. Oxidative stress in cardiovascular disease: a new avenue toward future therapeutic approaches. Recent Patents Cardiovasc Drug Discov 2006; 1:151-59

39. Schulz C, Konrad I, Sauer S, et al. Effect of chronic treatment with acetylsalicylic acid and clopidogrel on atheroprogression and atherothrombosis in ApoE-deficient mice in vivo. Thromb Haemost 2008; 99:190-95

40. Hankey GJ. Antiplatelet therapy for stroke prevention. Curr Atheroscler Rep 2007; 9:312-18 